\title{
Janus devant le miroir : Albert Sechehaye (1870-1946), linguiste théoricien face au grammairien praticien*
}

Pierre Swiggers

\section{(2) OpenEdition Journals}

\section{Édition électronique}

URL : https://journals.openedition.org/dhfles/3647

DOI : $10.4000 /$ dhfles.3647

ISSN : 2221-4038

\section{Éditeur}

Société Internationale pour l'Histoire du Français Langue Étrangère ou Seconde

\section{Édition imprimée}

Date de publication : 1 juin 2014

Pagination : 11-41

ISSN : 0992-7654

Référence électronique

Pierre Swiggers, « Janus devant le miroir : Albert Sechehaye (1870-1946), linguiste théoricien face au grammairien praticien* ", Documents pour I'histoire du français langue étrangère ou seconde [En ligne], 52 | 2014, mis en ligne le 02 août 2016, consulté le 28 mai 2021. URL : http://journals.openedition.org/ dhfles/3647 ; DOI : https://doi.org/10.4000/dhfles.3647

Ce document a été généré automatiquement le 28 mai 2021

(c) SIHFLES 


\title{
Janus devant le miroir : Albert Sechehaye (1870-1946), linguiste théoricien face au grammairien praticien*
}

\author{
Pierre Swiggers
}

À M. André Goosse, en hommage. «Mais d'abord le mécanisme de la langue n'est pas une chose si simple qu'on puisse espérer échapper à toute difficulté ; ordonner cette matière ne pourra jamais se faire sans quelque effort ; l'important, c'est que cet effort se produise dans la meilleure direction possible. [...]

Il va sans dire [...] que si ce travail de condensation et de raccourcissement était un

jour fait et bien fait pour notre langue, l'enseignement scolaire de la syntaxe en tirerait le plus grand avantage. Il en deviendrait plus solide, plus profitable pour l'esprit, plus facile

aussi. »

(Sechehaye 1916: 76)

\section{Une confrontation de vues : Chessex et Sechehaye}

Dans les années 1926-27, le journal suisse L'Éducateur - au sous-titre édifiant « Dieu Humanité - Patrie " - fut la scène d'une confrontation de vues, qui n'est pas sans rappeler de plus anciens différends entre grammairiens (-philosophes) et "grammatistes $~^{1}$, ni sans évoquer, de façon anticipative, ceux entre linguistes 
générativistes en quête d'adéquation explicative et grammairiens se confinant à l'adéquation « observationnelle et descriptive ».

Dans L'Éducateur du 18 septembre 1926 parut un article d'Albert Chessex, ancien instituteur et alors maître primaire supérieur à Lausanne ${ }^{2}$. Chessex y présentait trois ouvrages: une Introduction à l'étude de l'enseignement de la grammaire par A. Fontaine, Inspecteur général de l'Instruction publique ; La grammaire par les textes et par l'usage, de L. Poitrinal; enfin, un travail présenté comme «une grammaire originale» et intéressante, mais que Chessex n'hésite pas à critiquer sur plus d'un point.

Destinée à des élèves dont l'allemand est la langue maternelle, elle renferme quelques indications de détail à leur usage particulier. Mais elle conviendrait tout aussi bien à des Français ou à des Romands. Je me demande même si elle ne vaudrait pas mieux pour nos élèves du degré supérieur que la partie grammaticale de leur cours de langue. Je répondrais oui, si à côté d'avantages évidents, elle ne présentait pas quelques inconvénients sérieux. (Chessex 1926 : 270)

D'abord, l'auteur du compte rendu exprime son regret que l'ouvrage recensé ne renferme pas d'exercices - une critique qui est aussitôt rétractée par le recenseur, qui reconnaît, ou plutôt affirme, que le livre recensé se veut un « ouvrage théorique » (ce qui n'était pas tout à fait exact). Mais il énonce ensuite quelques réserves plus graves :

惯 L'ouvrage comporte(rait) une nomenclature «beaucoup trop compliquée et beaucoup trop savante. (Je serais curieux de voir comment les petits Zuricois [sic] vont s'y retrouver! [...] Il y a dans ce caractère trop savant du livre de M. Sechehaye un défaut regrettable. » (Chessex $1926: 270)$

5 䁂 L'ouvrage fourni(rai)t des explications trop abstraites : « Les élèves auront plus d'une fois quelque peine à les [= les explications abstraites] comprendre. De toute évidence, M. Sechehaye ne s'est pas mis à leur place. Il a écrit pour lui, spécialiste, et pour les adultes cultivés. C'est, dans un ouvrage d'enseignement, une faute qui n'est pas sans gravité. »(Chessex $1926: 270)$

6 匑 Certains points de doctrine sont inacceptables ou insuffisamment justifiés aux yeux du recenseur :

Quel avantage y a-t-il à subdiviser les pronoms personnels en deux catégories, les «pronoms conjoints sujets » et les «pronoms personnels absolus »? Est-il utile, à propos des compléments du verbe, de s'embarrasser d'un «complément partitif conjoint»? (Ex. Il en a fait douze, pour: Il a fait douze fautes). Est-il indispensable d'ajouter à la liste des propositions subordonnées les «propositions concessives de généralisation»? Et que dire du plaisir que réserve aux élèves le "prédicat psychologique »! Je pourrais multiplier les exemples. Mais je crois que vous êtes suffisamment édifiés. (Chessex 1926 : 270)

Chessex termine sa recension par la louange de certaines qualités, mais dans une lecture qui tiendrait compte des maximes conversationnelles de Grice, cette louange relève presque du sarcasme :

Mais il serait injuste de terminer l'examen de cette grammaire par des critiques, car elle a de solides qualités. Outre celles que nous avons déjà relevées, il faut en noter deux encore. C'est d'abord la forme matérielle du volume: couverture, reliure, papier, caractères typographiques, distribution des "blancs", tout est combiné pour plaire à l'œil et pour augmenter la clarté de l'ouvrage. C'est ensuite l'importance capitale que $\mathrm{M}$. Sechehaye, comme M. Poitrinal, donne à la conjugaison. Les 42 dernières pages de son livre lui sont consacrées (p. 77 à 118). Ici, nos critiques tombent: tout est clair, simple, pratique. Heureux les élèves qui auront entre les mains ces pages précieuses! Quant aux malheureux écoliers 
romands, ils ne possèdent pas même un paradigme de conjugaison. Ne dirait-on pas une gageure ?... (Chessex $1926: 270-271$ )

8 Face à ce compte rendu peu élogieux, l'auteur de l'ouvrage recensé, Albert Sechehaye, a préféré - en homme courtois - réagir en portant le débat à un niveau épistémologique, et en posant ainsi le problème du face-à-face entre pédagogue et linguiste. Dans sa réplique, intitulée "Le pédagogue et le linguiste ", Sechehaye répond d'abord au verdict négatif de Chessex à propos de la terminologie employée ${ }^{3}$ :

M. Chessex nous reproche d'avoir été, dans une grammaire destinée à des écoliers, trop compliqué et trop abstrait et d'avoir usé d'une terminologie parfois un peu rébarbative. Il est possible que nous ayons été plus compliqué et plus abstrait qu'on n'est accoutumé de l'être dans de tels manuels, il est possible que notre terminologie choque quelques habitudes, mais cela seul ne suffit pas à prouver que nous n'ayons pas de bonnes raisons pour avoir procédé de la sorte. Il s'agit en réalité d'un conflit entre le linguiste et le praticien de l'enseignement, deux hommes faits pour se comprendre mais qui ne s'entendent pas toujours parce qu'ils apportent au débat des préoccupations différentes. Quand il y a désaccord entre eux, il s'agit de savoir si c'est le linguiste qui ne tient pas compte des conditions de l'enseignement, ou si c'est le pédagogue qui ne sait pas apprécier la collaboration que lui offre l'homme de science. Pour nous, qui avons d'une part fait des problèmes de la grammaire théorique notre étude spéciale et qui, d'autre part, disposons d'une expérience pédagogique assez notable, nous avons cru pouvoir tenter la synthèse nécessaire entre les deux points de vue. (Sechehaye 1927 : 14)

Il prend soin de justifier aussi son point de vue en ce qui concerne la distinction, fonctionnelle et terminologique, du prédicat et de l'attribut, et à propos de l'importance attribuée à la bipolarité sujet-prédicat :

Ce terme [= prédicat] en effet est nécessaire pour nommer d'une façon générale la partie de la phrase qui s'oppose au sujet. L'usage scolaire français, qui consiste à nommer cette seconde partie de la phrase tantôt verbe (dans le pain nourrit), tantôt attribut (dans le pain est bon), est doublement fâcheux, d'abord parce qu'il voile ce qui fait l'unité de ces deux cas, ensuite parce qu'il exprime leur distinction par des termes hétéroclites (verbe est le nom d'une classe de mots et attribut celui d'une fonction logique). Or l'opposition du sujet et du prédicat, que notre terminologie met en évidence, est l'âme même de la phrase grammaticale et aussi le facteur psychologique essentiel dans le développement du discours vivant, qui est jusqu'à un certain point indépendant de la grammaire. Il en résulte qu'il y a lieu souvent de parler, à côté du sujet ou du prédicat grammatical, d'un sujet ou d'un prédicat psychologique. Et ces notions sont des clefs aussi simples qu'efficaces pour expliquer (c'est-à-dire expliquer vraiment, classer, définir, faire comprendre) certains faits de grammaire et de style. (Sechehaye $1927: 15-16)$

Or, chose intéressante, le " conflit entre le linguiste et le praticien de l'enseignement " ${ }^{4}$ qu'évoque Sechehaye n'opposait pas seulement deux hommes (en l'occurrence Sechehaye et Chessex), ou deux types de figures (linguiste et didacticien) : il répondait aussi à une tension (subliminale, peut-être ?) entre deux aspects - ou si l'on veut, deux pulsions - dans l'activité de Sechehaye : la tension entre l'effort de théorisation dans la tête du linguiste, d'un côté, et l'attente de résultats concrets dans la pratique langagière des élèves, de l'autre. On sait qu'il y a de meilleurs mariages.

11 Afin de jeter un éclairage sur ce problème d'historiographie, il convient de se tourner vers le parcours professionnel et intellectuel de l'homme ${ }^{5}$ et l'élaboration de son œuvre ${ }^{6}$. 


\section{Albert Sechehaye : un linguiste « constructiviste»}

À distance, la carrière d'Albert Sechehaye (= Charles Albert Sechehaye, 1870-1946) semble conjuguer un versant "Jekyll» et un versant "Hyde »". Ayant reçu une formation philologique (en lettres classiques) à Genève, Sechehaye devient précepteur privé et, à partir de 1894, après un séjour d'études à Leipzig, lecteur de français, en Bohème (à Aussig-sur-Elbe), de 1894 à 1897, et à Göttingen, de 1897 à 1901. Pendant cette période de lectorat il prépare une thèse de morphosyntaxe historique du français, thèse qu'il soutient le 3 mars $1902^{8}$. De retour à Genève, Sechehaye redevient précepteur privé, mais en 1903 il est nommé maître de conférences au "Séminaire de français moderne ». C'est dans l'entourage stimulant de Ferdinand de Saussure, chez qui il avait suivi en 1891-93 des cours de sanskrit, mais dont il ne suivra pas l'enseignement en linguistique générale ${ }^{9}$, que Sechehaye va élaborer une œuvre de linguiste théoricien et, davantage, d'épistémologue de la linguistique. En effet, en 1908 paraît son premier livre Programme et méthodes de la linguistique théorique. Ce travail, que grammairiens (H. Yvon), linguistes (A. Meillet), philosophes (A. Naville), psycholinguistes (A. Grégoire), philologues (K. Vossler) et phonologues (R. Jakobson) ${ }^{10}$ saluent comme innovateur, se propose de poser les fondements de la linguistique théorique, une science qui prend comme objet le phénomène du langage " pris dans son idée abstraite » (Sechehaye $1908: 9$ ).

Dès les années 1910, Sechehaye, fort de son expérience de théoricien et de linguiste diachronicien (en 1909 il publie une petite grammaire historique du français), s'engage dans la voie de la grammaire synchronique à visée didactique. Il conjuguera ainsi un important effort de réflexion théorique avec un investissement dans la description grammaticale "pédagogique »; un bref relevé de ses principales publications entre Programme et méthodes et l'Abrégé de grammaire française permet de dégager ce double axe.

1. 1908. Programme et méthodes de la linguistique théorique. Psychologie du langage. Paris : Champion.

2.1914. "Les règles de la grammaire et la vie du langage", Germanisch-romanische Monatsschrift, 6, 288-303 et 341-351.

3. 1916. «La méthode constructive en syntaxe », Revue des Langues romanes, 59, 44-76.

4. 1916. Édition, avec Charles Bally, de : Ferdinand de Saussure, Cours de linguistique générale. Lausanne/Paris : Payot.

5. 1916. Collaboration à H. Hoesli, Éléments de langue française. Zürich : Erziehungsdirektion.

6. 1917. "Les problèmes de la langue à la lumière d'une théorie nouvelle", Revue philosophique de la France et de l'étranger, 42, 1-30.

7. 1920. «Les deux types de la phrase ». In Mélanges d'histoire littéraire et de philologie offerts à M. Bernard Bouvier à l'occasion du XXXe anniversaire de sa nomination comme Professeur ordinaire à la Faculté des Lettres de l'Université de Genève, 315-322.

8. 1926. Essai sur la structure logique de la phrase. Paris : Champion.

9. 1926. Abrégé de grammaire française sur un plan constructif suivi d'un tableau systématique des conjugaisons. Zürich : Verlag der Sekundarlehrerkonferenz des Kantons Zürich.

10. 1926. Le verbe français. Tableau systématique de ses conjugaisons. Zürich: Verlag der Sekundarlehrerkonferenz des Kantons Zürich ; Genève : Eggimann. [Publication séparée des p. 78-118 de l'Abrégé ; réédition en 1932] 
En octobre 1912 il fait une conférence à la Société des romanistes de Zurich, dans laquelle il expose ses premières idées sur la description de la syntaxe du français moderne. Ce travail, dans lequel il définit la grammaire comme l'organisation de tous les faits d'expression conventionnelle ${ }^{11}$, est centré sur la description de l'article défini. Il sera suivi d'une étude beaucoup plus ambitieuse que Sechehaye publie dans la Revue des langues romanes: «La méthode constructive en syntaxe ». Ce travail, paru la même année que l'édition du Cours de linguistique générale ${ }^{12}$, propose un véritable programme de linguistique constructive dans une perspective théorique et didactique. Il convient de l'analyser plus en détail, vu qu'il définit les fondements sur lesquels sera érigé tout le travail grammatical de Sechehaye.

\section{L'élaboration d'un plan de construction " méthodique »}

Sechehaye commence par souligner la nécessité d'une méthode rigoureuse, à la fois pour l'approche scientifique et pour l'enseignement d'une langue: «La question des méthodes d'enseignement en matière de langue, et principalement en ce qui concerne la langue maternelle, n'est pas seulement du ressort des pédagogues; elle a aussi un aspect proprement scientifique et linguistique. » (Sechehaye $1916: 44$ )

Anticipant l'article pionnier de Tesnière (1934; cf. Swiggers 1994), il préconise une approche systémique de la syntaxe: celle-ci doit «rendre compte» de la forme abstraite (= les principes d'organisation hiérarchique et linéaire) des phrases :

L'esprit de la syntaxe, pour ainsi dire, doit être constructif et architectural. Ce que cette science demande, par sa nature même, ce n'est pas une énorme et savante compilation de faits superficiellement classés, mais un substantiel et lumineux raccourci de ces faits. Notre parole est faite, avec l'apport personnel que nous y ajoutons, d'un immense matériel d'unités significatives que la langue nous apporte rangées selon certains principes de construction et d'ordonnance qui sont comme le moule et la forme abstraite de toutes nos phrases. C'est de cette forme abstraite que la syntaxe doit nous rendre compte. (Sechehaye $1916: 76$ )

Si l'auteur reconnaît que dans la conception de la démarche générale il y a deux options fondamentales ${ }^{13}$ - qu'on appellerait aujourd'hui 'sémasiologique' et 'onomasiologique' -, il souligne qu'il est impossible, au plan de l'objet étudié, de dissocier la « pensée » et la « forme » dans la langue : «Le fait de langue consiste exclusivement en la rencontre d'une valeur conceptuelle (une idée) et d'une valeur matérielle (l'idée de certains sons), lesquelles n'existent d'ailleurs que par leur union et la détermination que leur donne l'ensemble du système dont elles font partie. » (Sechehaye 1916 : 46)

En quoi consiste alors cette "méthode constructive " que Sechehaye veut élaborer? L'auteur fait remarquer d'abord que cette méthode a été illustrée par des précurseurs, à la fois en didactique et en description grammaticale de langues. Il cite deux exemples : pour la didactique du français, son compatriote le père Grégoire Girard ${ }^{14}$, et pour la description grammaticale et linguistique (en l'occurrence de l'allemand), Ludwig Sütterlin.

En proposant cette systématisation non seulement nous ne faisons que continuer le mouvement inauguré par le père Girard, mais encore nous nous trouvons en harmonie avec le progrès qui se dessine actuellement dans la science de la syntaxe. Qu'on prenne par exemple l'ouvrage de Sütterlin (Die deutsche Sprache der Gegenwart), et on y trouvera dans la dernière partie un plan constructif ressemblant 
par certains traits généraux et par plusieurs détails au nôtre. En se faisant constructive la syntaxe obéit à une véritable nécessité qui sera toujours plus sentie. Cependant nous ne croyons pas qu'un essai d'application conséquente et fondé sur des raisons de principe ait encore été tenté, et c'est ce qui nous autorise à présenter le nôtre. (Sechehaye $1916: 50$ )

19 Comment la méthode constructive en syntaxe s'articule-t-elle? Elle procède (contrairement à la décomposition descendante selon la double articulation) par étapes de complexité ascendante. Le point de départ est la construction minimale et complète, nécessaire pour énoncer un fait (structure Sujet-Prédicat); celle-ci est constituée de deux «termes » : sujet (substantif + «les particules et les mots auxiliaires qui lui sont propres ») et le verbe prédicat. Sur cette construction minimale se greffent les procédés d'extension, par l'ajout de compléments qui peuvent se joindre au sujet et au verbe : complément attributif - adjectif rattaché au prédicat - complément de relation complément (attributif / de relation) d'un complément de relation. Ensuite il y a deux ordres de complexification du noyau SP avec ses compléments :

\section{l'occurrence) ${ }^{16}$ :}

Dans le domaine des subordonnées on verra que les diverses espèces de propositions présentent un habitus grammatical différent selon les attaches logiques et grammaticales qu'elles peuvent avoir avec les diverses parties de la proposition simple : il y en a qui sont assimilables à des compléments attributifs, qui vont de pair avec l'adjectif et le participe, d'autres qui tiennent du substantif; elles sont sujet, apposition, complément de relation ou de comparaison. Il y a là les éléments d'une classification constructive, c'est même un des points sur lesquels la méthode que nous préconisons est déjà plus ou moins entrée dans la pratique (Sechehaye 1916:62); méthodologique entre synchronie et diachronie ${ }^{17}$; seulement, Sechehaye fait observer qu'entre histoire et système, il y a une analogie et que tout "système " linguistique n'est qu'une coupe (" hypostasiante ») dans un continuum fluctuant. "Le rapport dans le temps sans se confondre avec le rapport grammatical lui ressemble et contribue à l'éclairer "; "À cette inconsistance dans le temps correspond un état perpétuel d'inachèvement et de provisoire dans chaque équilibre momentané du système.» (Sechehaye 1916:63 et 64)

Le rôle du grammairien constructiviste est d'observer les faits de langue, de les réduire à une structure, en opérant des classifications fonctionnelles. Cette classification doit combiner des aspects formels, fonctionnels et sémantiques. Ainsi Sechehaye intègre-t-il le paramètre du "degré de synthèse" (au sens de Sapir 1921) pour justifier la séparation entre prépositions fortes et prépositions faibles. Les prépositions fortes sont celles «dont la valeur générale est relativement bien déterminée » (Sechehaye 1916: 55) : il s'agit de prépositions ayant un sens lexical ou lexicalement descriptible (" avec », "sans »). Les prépositions faibles sont celles "dont la synthèse psychologique et grammaticale est plus complète » («de», «à »).

Documents pour l'histoire du français langue étrangère ou seconde, 52 | 2014 
Mais le grammairien constructiviste doit aussi prendre des décisions «normatives »; par ce terme, Sechehaye entend, non pas des verdicts puristes ou prescriptifs, mais des fixations systématisantes ou stabilisantes (ou pourrait même dire : stochastiques) :

Notre science, bien loin d'ignorer les innombrables exceptions dont la langue fourmille et tous les caprices individuels dont la parole est le théâtre, veut fournir ce qui est nécessaire à leur appréciation. Elle sait que tout cela, en vertu d'une nécessité immanente, se groupe autour d'un système normatif, un peu incertain par endroits, un peu flottant, mais qui tend toujours à se constituter [sic]. Elle agit donc dans l'esprit même de la langue lorsqu'elle esquisse ce système et lui prête ici ou là des contours assez précis pour pouvoir le dessiner. On peut poser en fait que les sujets parlants ne restent pas dans le vague. Toutes les fois qu'ils construisent, ils suivent une analogie quelconque. Ce qui produit l'incertitude, c'est qu'un même élément, par exemple notre participe présent, d'une occasion à l'autre est balancé entre deux analogies diverses. Le grammairien étend à la théorie le procédé de la pratique : consultant au plus près sa conscience de sujet parlant, il fixe son choix et il attribue au choix fait une valeur normative, une certaine stabilité dans son système. (Sechehaye 1916 : 65-66)

\section{Résumons : Quelles sont les caractéristiques de cette méthode constructive?}

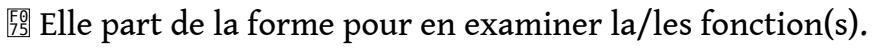

医 Elle est « ascendante » (ou « graduée »).

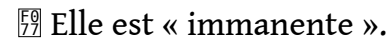

䢙 Elle est « flexible » ${ }^{18}$.

㗜 Elle détermine son champ de manière stricte et lui assigne une structuration (hiérarchique) interne :

La syntaxe telle que nous l'entendons n'épuise pas l'étude de la langue. Bien au contraire elle a ceci pour elle qu'elle retient et isole ce qui est proprement de son ressort, et qu'elle empêche cette partie de la grammaire d'aller se perdre en dehors de son domaine pour s'égarer dans une infinité de détails étranges ou superflus. Étant donné un problème nouveau qu'elle aborde, elle sait exactement comment elle doit le traiter, ce qu'elle doit en dire et ce qu'elle doit laisser de côté. Elle ne procède pas par association d'idées, épuisant de proche en proche tout ce qu'on peut constater à propos d'un objet proposé à son examen. Elle ne s'attache qu'à ce qui peut et doit entrer dans sa construction.

Cette construction se compose en réalité d'une série de petites syntaxes particulières, quelquefois juxtaposées, le plus souvent superposées et se portant les unes les autres comme les pierres de taille dans un mur. Un type syntaxique initial ou nouveau étant posé, il faut énoncer toutes les règles auxquelles il donne lieu, tout ce qui dans le jeu possible de cet organe syntaxique est incontestablement fait de langue, norme de construction dans la parole. (Sechehaye $1916: 72-73$ )

\section{La transposition ("translation ») vers la pratique: l'Abrégé}

33 C'est dans la continuité directe de ce programme « constructiviste » que s'échelonnent les travaux de grammaticographie pratique et didactique de Sechehaye. En 1918, il collabore aux Éléments de langue française de Hans Hoesli, et si en 1920 il publie dans les Mélanges Bouvier une étude plutôt théorique ${ }^{19}$, il poursuit sa réflexion sur l'enseignement de la grammaire comme le montre le texte achevé, soigneusement rédigé, de l'Abrégé de grammaire française. 
Ce petit ouvrage est destiné à accompagner le Cours pratique de langue française publié par M. Hoesli et qui, faisant suite à ses Éléments de langue française, correspond à la troisième et dernière année d'enseignement dans les écoles secondaires du canton de Zurich.

Comme M. Hoesli l'a rappelé dans l'introduction de son Cours pratique, nous avons écrit pour lui une courte grammaire systématique qui a figuré dans la $2^{\mathrm{e}}$ édition de ses Éléments. Lorsqu'il nous demanda, il y a longtemps déjà, de composer un texte nouveau et plus complet pour le manuel de troisième année qu'il projetait, nous avons accepté à condition qu'il nous autorisât à le disposer sur un plan nouveau et rigoureusement constructif. (Sechehaye 1926a : VII)

L'Abrégé de Sechehaye peut être considéré, à mon avis, comme une des meilleures synopses grammaticales du français. Cela tient en premier lieu au fait que l'auteur a élaboré sa grammaire selon un plan bien pensé et pesé. L'importance d'un plan nettement articulé est d'ailleurs mise en évidence par l'auteur lui-même :

Le problème du plan n'est d'ailleurs pas le seul qui nous ait occupé pendant la rédaction de notre opuscule. Toutes les questions générales de théorie grammaticale ont été également envisagées. On verra en particulier que nous avons partout distingué avec soin ce qui est phonique et appartient en propre à la langue, de ce qui est orthographique et ne regarde que la convention de l'écriture. Nous avons aussi mis à part et signalé par une distinction typographique tout ce qui dans la grammaire est irrégulier, locutionnel et proprement lexicologique. Pour d'autres questions plus délicates nous renvoyons à quelques notes que nous avons mises au bas des pages et qui, sans surcharger l'exposé, permettront au maître de comprendre certains principes généraux dont nous nous sommes inspiré. En grammaire, comme dans toute autre science, la clarté de l'exposé, la solidité des doctrines et l'intérêt de l'enseignement dépendent de la justesse des notions fondamentales. On enseigne mieux, avec plus de conviction et plus d'assurance, quand on se sent sur un terrain solide. (Sechehaye 1926a : IX)

Cette préoccupation "méthodique» et théorique, outre qu'elle repose sur un tempérament de théoricien, a aussi des raisons «contextuelles ». En effet, l'Abrégé de Sechehaye qui, rappelons-le, accompagne un manuel didactique et qui met en pratique un «programme » constructiviste envisagé par l'auteur dès les années 1910, s'insère dans une double évolution de la grammaticographie française à l'époque : d'une part, une évolution marquée par les discussions, autour des années 1910, concernant la réforme de l'enseignement grammatical - réforme affectant l'orthographe ( $c f$. les travaux de Clédat et l'information fournie dans sa Revue de philologie française), la nomenclature ( $c f$. Sudre 1906), et le traitement de la syntaxe; d'autre part, une évolution marquée par le renouveau "stylistique» (Bally 1905) et "psychosociologique " (Brunot 1922) en grammaire. Signalons ici quelques textes-clés (cf. Swiggers 2004, 2012) :

1. 1905. Ch. Bally: Précis de stylistique. Esquisse d'une méthode fondée sur l'étude du français moderne.

2. 1905-1908. F. Brunot \& N. Bony : Méthode de langue française.

3. 1907. Ch. Maquet: Rapport présenté au Conseil supérieur de l'Instruction publique au nom de la Commission chargée d'étudier la simplification des nomenclatures grammaticales.

4. 1906. L. Sudre: «Des nomenclatures grammaticales». In Conférences au Musée pédagogique. L'enseignement de la grammaire, 101-128.

5. 1909. Ch. Bally : Traité de stylistique française.

6. 1909. F. Brunot \& N. Bony: L'enseignement de la langue française : ce qu'il est, ce qu'il devrait être dans l'enseignement primaire. 
7. 1909. F. Brunot \& Ch. Maquet: «Simplification et unification des nomenclatures grammaticales ». Revue universitaire, 8, 340-354.

Il n'en reste pas moins que dans les grammaires françaises publiées entre 1920 et 1926 ce (double) renouveau ${ }^{20}$ n'est pas concrétisé de manière uniforme, ni avec la même intensité. Il suffirait de comparer par exemple les grammaires suivantes ${ }^{21}$ en ce qui concerne (a) l'architecture générale de la grammaire; (b) la reconnaissance de constituants de phrases ; (c) l'emploi fait des «parties du discours » traditionnelles (et leur définition); (d) le rôle accordé à des données diachroniques; (e) l'importance donnée à l'orthographe ; (f) la définition et l'articulation de la partie de syntaxe, etc.

1. 1921. M. Lanusse \& H. Yvon: Cours complet de grammaire française. À l'usage de l'enseignement secondaire classique. Grammaire et exercices. Grammaire complète. Classes de grammaire et classes supérieures.

2.1921. F. Strohmeyer: Französische Grammatik auf sprachhistorisch-psychologischer Grundlage.

3. 1922. F. Brunot : La pensée et la langue. Méthode, principe et plan d'une théorie nouvelle du langage appliquée au français.

4. 1922. R. Radouant: Grammaire française.

5. 1923. B. Van Hollebeke \& O. Merten (révision par J. Fleuriaux) : Grammaire française à l'usage des Athénées, des Collèges et Écoles moyennes.

6. 1926. Th. Engwer \& E. Lerch : Französische Sprachlehre.

Passant à une analyse interne de l'ouvrage de Sechehaye, que peut-on dire, comme appréciation globale, de l'Abrégé ? Nous nous bornerons à traiter les points suivants :

Organisation de l'ouvrage : l'Abrégé présente une structure limpide (cf. la macrostructure reproduite dans l'Annexe I) : elle comporte six parties, chacune divisée en chapitres, et les chapitres sont à leur tour divisés en paragraphes numérotés. On signalera aussi la présence, à la fin de l'ouvrage (Sechehaye 1926a: 73-76), d'un Index «par ordre des matières ", c'est-à-dire selon l'ordre d'apparition de termes et de notions dans le texte même de la grammaire.

Démarche didactique : la grammaire procède en rapport direct avec la structuration de la matière linguistique et cette suite ascendante, correspondant à une progression dans l'apprentissage de la langue, est reflétée dans la complexité croissante des phrases-types qui se trouvent au début de chaque partie et qui sont à la fois des points d'ancrage pour la description grammaticale (et sa terminologie), des repères mnémotechniques et des modèles d'imitation. Nous avons reproduit dans l'Annexe II l'ensemble des phrasestypes qu'on trouve dans l'Abrégé.

Bally et Sechehaye ont mis en évidence le rôle (théorique et descriptif) crucial de ces phrases-types : « La méthode constructive que M. Sechehaye a définie et décrite (M.C.) et dont il a tenté une première réalisation dans un manuel scolaire [...], croit pouvoir résoudre ce problème, en étudiant les divers types de la phrase et en procédant du simple au complexe. En effet, l'entité syntaxique essentielle n'est ni le mot, ni sa catégorie, ni sa forme, mais la phrase-type où le mot est appelé à jouer un rôle. La tâche de la syntaxe consiste donc à passer en revue, dans un ordre méthodique, les divers schémas de la phrase. » (Bally et Sechehaye $1928: 51$ )

Les phrases-types nous semblent présenter plusieurs caractéristiques intéressantes :

[5] $\mathrm{Au}$ point de vue linguistique, 

(ce qui permet à l'auteur d'introduire la notion de "complément de relation ") et la progression vers l'unité propositionnelle. À ce dernier niveau, la distinction fondamentale est celle entre proposition simple/indépendante et proposition complexe. On notera aussi que dans cette grammaire constructive, l'écrit n'est pas perdu de vue (voir par ex. Sechehaye 1926a : 2 et 12, et $c f$. la présence de deux sections sur la ponctuation, dans les parties $\mathrm{V}$ et $\mathrm{VI})$.

51 Points saillants: à côté de l'emploi judicieux de phrases-types, on doit mentionner comme points saillants de la description grammaticale, la théorie du prédicat (et l'emploi de la notion de prédicat psychologique, Sechehaye 1926a : 60), la distinction (tout à fait justifiée) entre pronoms conjoints et pronoms absolus (Sechehaye 1926a : 18, 23), et la distinction entre prépositions fortes et faibles (Sechehaye 1926a: 27 ; voir aussi ibid., p. 14 et p. 24).

é de Sechehaye se présente ainsi comme une grammaire à fondement théorique et à usage didactique, comme une description systématisante, qui distingue (et met toujours adéquatement en corrélation) forme, valeur et emploi, et comme un guide pour la maîtrise progressive du spectre sémantico-syntaxique et pragmatico-discursif de la langue.

\section{Conclusion}

(1) D'abord, le fait que la "tension » entre le côté «linguiste théoricien » et le côté "grammairien pédagogue " a été, chez Sechehaye, une tension harmonieuse ${ }^{22}$ et de longue durée ${ }^{23}$. Si son goût de la linguistique théorique a sans doute été éveillé par l'enseignement de Saussure ( $c f$. Sechehaye 1915) et stimulé, ensuite, par son séjour à Leipzig et par l'influence de son collègue Bally, on sait aussi que son intérêt pédagogique a été une vocation précoce : 
C'est une vocation qui m'est venue un jour que je considérais avec mélancolie un petit manuel très quelconque de grammaire française, dont, maître débutant dans une école de commerce, je m'étais engagé à inculquer le contenu à une classe de jeunes gens de langue allemande. Je me demandais ce que signifiaient toutes les prescriptions formelles qui y étaient doctoralement énoncées et je conçus l'ambition de comprendre un jour ce qu'il y avait derrière tout cela afin de pouvoir mieux l'enseigner moi-même et peut-être enseigner aux autres à l'enseigner. (Sechehaye $1933: 13)^{24}$ et dans les publications de Sechehaye, et celui-ci n'a jamais caché son attachement au noble but de l'enseignement des langues : "Puisse notre petit travail être utile à la jeunesse et à tous ceux qui ont la tâche belle et ardue de l'instruire. Les idées qui sont à la base ont été soigneusement pesées et l'exécution, qui ne devait pas les trahir, a été longuement mûrie. Nous serons amplement récompensé de nos efforts si le corps enseignant du canton de Zurich accueille notre tentative avec intérêt. » (Sechehaye 1926a: X)

(2) En deuxième lieu, il convient de souligner que l'auteur, dans la «transposition » de la théorie à la pratique, a fourni des apports sinon innovateurs, du moins très estimables ; ainsi, dans l'Abrégé, Sechehaye propose une description

(a) qui suit la complexité croissante de l'expression langagière et de la construction de la phrase ;

(b) qui combine l'examen de la forme avec celui de la fonction ;

(c) qui sépare des séries formellement identiques ou semblables, mais fonctionnellement divergentes.

Autant d'initiatives qui distinguent l'Abrégé de la plupart des grammaires pratiques du français alors en usage. Pour cela, il fallait en effet repenser les plans traditionnels et montrer un peu d'audace terminologique.

Voilà à quoi se bornent nos abstractions et nos complications et, quand elles seraient plus graves, nous croyons que l'enseignement grammatical a tout à gagner à être scientifique, soit que dans les classes supérieures on présente aux élèves les grands faits généraux de la grammaire, soit qu'au degré inférieur on laisse la théorie au maître et qu'on se contente d'user d'une terminologie et de formulations vraiment correctes. Les maîtres de mathématiques, de chimie, de géologie, etc., ne se font pas faute d'introduire dans leurs exposés les idées assez abstraites et souvant [sic] aussi la terminologie barbare de leur discipline. Ils abusent parfois d'un droit bien naturel que personne ne leur conteste : celui de prendre la science elle-même comme base et comme point de départ de leur enseignement. Qui osera refuser ce droit au grammairien le jour où, las des à peu près de la routine, il s'avisera de le revendiquer? Puisse ce jour venir bientôt, car c'est alors seulement que la grammaire sortira du discrédit dans lequel elle végète à l'école et par sa propre faute. Comment une doctrine qui ne dit rien de solide à l'esprit et que le pédagogue enseigne parce que c'est celle du livre, pourrait-elle captiver l'esprit des enfants? Mais quand le maître comprendra et pourra faire comprendre les phénomènes du langage, on le verra prendre du plaisir à son enseignement grammatical et ses élèves en prendront aussi... même en usant du terme affreux de "prédicat psychologique ». (Sechehaye $1927: 16$ )

61 (3) En troisième lieu, dans son Abrégé Sechehaye a su brider ses envolées théorisantes afin de présenter, avec un minimum de théorie, une description grammaticale accessible à des élèves du secondaire et acceptable pour des grammairiens à l'esprit ouvert. Henri Yvon était parmi ces derniers ${ }^{26}$ : 
Rédigeant une grammaire élémentaire, M. Sechehaye, à la fois linguiste et pédagogue, s'est placé à un point de vue tout différent ; il est parti de l'observation des faits du langage, et de ce fait fondamental que nous ne parlons pas par mots, mais par groupes de mots. Or, dans la frase française actuelle, plus que dans d'autres formes de frase, les mots se groupent autour de deus centres d'attraction, le nom d'une part, le verbe d'autre part. Donnant au mot sintaxe un sens plus général que celui que lui donnent habituellement nos grammaires, et plus voisin $\mathrm{du}$ sens étimologique, M. Sechehaye avait déjà exposé en 1916 une métode de sintaxe constructive. Il l'a appliquée dans cet abrégé où il étudie séparément le groupe nominal et le groupe verbal : il réalise ainsi le tipe de grammaire française que $\mathrm{M}$. Meillet a préconisé récemment dans une conférence faite à la Société de pédagogie de Paris. Cette disposition entraîne des redites, mais M. Sechehaye a préféré un plan conforme aus faits à une construction tout à fait logique. Ayant pris autrefois, dans des circonstances analogues, la même décision, nous ne songeons pas à l'en blâmer. (Yvon 1927 : 146)

L'œuvre grammaticale de Sechehaye devrait d'ailleurs laisser son empreinte sur les travaux de Cornelis de Boer (Introduction à l'étude de la syntaxe française, 1933) et de Georges Galichet (Essai de grammaire psychologique du français moderne, 1947). Et pourtant ${ }^{27}$... Comme on l'a vu, l'Abrégé ne fut pas accueilli sans réserves ou critiques par les pédagogues et les praticiens de l'enseignement grammatical, et la prompte réaction de Sechehaye dans L'Éducateur laisse deviner la déception qu'il en a éprouvée ${ }^{28}$.

\section{BIBLIOGRAPHIE}

AN. (1944). « Publications linguistiques de M. Albert Sechehaye ». Cahiers Ferdinand de Saussure, 4, 3-5.

AN. (1945). « Nécrologie : Albert Sechehaye ». Cahiers Ferdinand de Saussure, 5, 53.

BALLY, Charles, SECHEHAYE, Albert (1928). «Quelles sont les méthodes les mieux appropriées à un exposé complet et pratique de la grammaire d'une langue quelconque? ». In Actes du Premier Congrès international de linguistes à La Haye du 10 au 15 avril 1928. Leyde : A.W. Sijthoff, 36-53.

BRUNOT, Ferdinand (1920-21). « Le renouvellement nécessaire des méthodes grammaticales ». Revue universitaire, 29/2, 161-178 et 30/1, 21-39.

CHESSEX, Albert (1926). « Pour l'enseignement du français ». L'Éducateur, 62, n du 18 septembre 1926, 265-271.

CHESSEX, Albert (1927). [réponse à A. Sechehaye]. L'Éducateur, 63, nº du 8 janvier 1927, 16.

CHEVALIER, Jean-Claude (1990). «Syntaxe et sémantique en grammaire. Histoire d'une méprise : Ferdinand Brunot et Charles Bally ». In Sprachtheorie und Theorie der Sprachwissenschaft. Geschichte und Perspektive. Festschrift für Rudolf Engler zum 60. Geburtstag. Tübingen : G. Narr, 95-107.

FORSTER, Simone (2004). « Les visionnaires du début du XX siècle ». Bulletin CIIP, 14 (mars 2004), $2-3$.

FRYBA-REBER, Anne-Marguerite (1994). Albert Sechehaye et la syntaxe imaginative. Contribution à l'histoire de la linguistique saussurienne. Genève : Droz. 
FRYBA-REBER, Anne-Marguerite (1995-96). « Charles-Albert Sechehaye : un linguiste engagé ». Cahiers Ferdinand de Saussure, 49, 123-137.

GRÉGOIRE, Antoine (1927). C.r. de Sechehaye 1926a. Revue belge de philologie et d'histoire, 6, 313.

JAKOBSON, Roman (1939). C.r. de N. van Wijk, Phonologie, een hoofdstuk uit de structurele taalwetenschap. Acta Linguistica, 1, 123-129.

LAUWERS, Peter (2004). La description du français entre la tradition grammaticale et la modernité linguistique. Étude historiographique et épistémologique de la grammaire française entre 1907 et 1948. Leuven/Paris : Peeters.

LÁZARO CARRETER, Fernando (1947). « Albert Sechehaye (1870-1946) ; Charles Bally (1865-1947) ». Revista de filología española,31-32, 406-409.

MEILLET, Antoine (1908). C.r. de Sechehaye 1908. Bulletin de la Société de Linguistique de Paris, 15, xxiij-xxv.

MEILLET, Antoine (1921). C.r. de Sechehaye 1920. Bulletin de la Société de Linguistique de Paris, 22, 171-173.

MEILLET, Antoine (1926). C.r. de Sechehaye 1926b. Bulletin de la Société de Linguistique de Paris, 27, $1-2$.

NAVILLE, Adrien (1908). « Le programme et les méthodes de la linguistique théorique. À propos d'un ouvrage récent ». Revue de synthèse historique, 1908, 299-304.

NIEDERLÄNDER, Helmut (1981). Französische Schulgrammatiken und schulgrammatisches Denken in Deutschland von 1850 bis 1950. Frankfurt : Lang.

REVERDIN, Henri (1959). « Albert Sechehaye (1870-1946) ». In Histoire de l'Université de Genève. Annexes : Historique des Facultés et des Instituts, 1914-1956. Genève : Georg, 109-111.

SAPIR, Edward (1921). Language. New York : Harcourt \& Brace.

SECHEHAYE, Albert (1905). «L'imparfait du subjonctif et ses concurrents dans les hypothétiques normales en français. Esquisse de syntaxe historique ». Romanische Forschungen, 19/2, 321-406.

SECHEHAYE, Albert (1908). Programme et méthodes de la linguistique théorique. Psychologie du langage. Paris : Champion.

SECHEHAYE, Albert (1914). « Les règles de la grammaire et la vie du langage ». Germanischromanische Monatsschrift, 6, 288-303 et 341-351.

SECHEHAYE, Albert (1915). « Leçon du 28 février 1913 à l'Université de Genève ». In Ferdinand de Saussure (1857-1913). Plaquette d'hommages. Genève : Sonor, 59-67.

SECHEHAYE, Albert (1916). « La méthode constructive en syntaxe ». Revue des langues romanes, 59, 44-76.

SECHEHAYE, Albert (1917). «Les problèmes de la langue à la lumière d'une théorie nouvelle ». Revue philosophique de la France et de l'étranger, 42, 1-30.

SECHEHAYE, Albert (1920). « Les deux types de la phrase ». In Mélanges d'histoire littéraire et de philologie offerts à M. Bernard Bouvier à l'occasion du XXXe anniversaire de sa nomination comme Professeur ordinaire à la Faculté des Lettres de l'Université de Genève. Genève : Sonor, 315-332.

SECHEHAYE, Albert (1926a). Abrégé de grammaire française sur un plan constructif suivi d'un tableau systématique des conjugaisons pour servir à l'étude du Cours pratique de langue française de Hans Hoesli. Zürich : Verlag der Sekundarlehrerkonferenz des Kantons Zürich. 
SECHEHAYE, Albert (1926b). Essai sur la structure logique de la phrase. Paris : Champion.

SECHEHAYE, Albert (1927). « Le pédagogue et le linguiste ». L'Éducateur, 63, nº du 8 janvier 1927, $14-16$.

SECHEHAYE, Albert (1930). « La méthode constructive en grammaire et son application ». In 59. Jahrbuch des Vereins schweizerischer Gymnasiallehrer, 106-108. [Réimpression dans Cahiers Ferdinand de Saussure, 4, 1944, 22-25]

SECHEHAYE, Albert (1933). « La pensée et la langue, ou comment concevoir le rapport organique de l'individuel et du social dans le langage ? ». Journal de psychologie normale et pathologique, 30, 57-81. [Réimpression dans Cahiers Ferdinand de Saussure, 4, 1944, 26-52]

SECHEHAYE, Albert (1941). « Les classes de mots et l'imagination ». Cahiers Ferdinand de Saussure, $1,77-88$.

SWIGGERS, Pierre (1984). « La systématicité interne de la langue: Deux axes dissociés ou leur combinaison?». Travaux de Linguistique et de Littérature,22, 65-70.

SWIGGERS, Pierre (1992). « La grammaire des Académiciens prise d'assaut : un exemple de 'récurrence différentielle' dans l'histoire de la grammaire française ». Travaux de Linguistique et de Philologie, 30, 125-137.

SWIGGERS, Pierre (1994). « Aux débuts de la syntaxe structurale : Tesnière et la construction d'une syntaxe ». In Mélanges Lucien Tesnière (= Linguistica, 34/1) [Ljubljana], 209-219.

SWIGGERS, Pierre (2004). « Aux côtés d'Arsène Darmesteter : Léopold Sudre ». Revue des Langues romanes, 108, 483-503.

SWIGGERS, Pierre (2012). « Les combats de Clédat. Étapes d'une 'linguistique française' en élaboration, selon la voie (voix ?) de la grammaire ». In Actes du $3^{e}$ Congrès Mondial de Linguistique française. [Édition sur CD-ROM, p. 109-126. SHSWeb of Conference 1(2012). DOI. 10.1051/shsconf/ 2012à100343]

TESNIÈRE, Lucien (1934). « Comment construire une syntaxe ». Bulletin de la Faculté des Lettres de Strasbourg, 7, 219-229.

VOSSLER, Karl (1908). C.r. de Sechehaye 1908. Deutsche Literaturzeitung, 20, 1240-1246.

WIBLÉ, Eugène (1946-47). « Charles Bally, 4 février 1865/10 avril 1947 ; Albert Sechehaye, 4 juillet 1870/2 juillet $1946 »$. Vox Romanica, 9-10, 107-109.

YVON, Henri (1905). C.r. de Sechehaye 1905. Revue de Philologie française, 19, 300-305.

YVON, Henri (1908). Cr. de Sechehaye 1908. Revue de Philologie française, 22, 70-73.

YVON, Henri (1927). C.r. de Sechehaye 1926a. Revue de Philologie française, 39, 144-149.

\section{ANNEXES}

\section{Annexe I}

Le plan général de l'Abrégé de grammaire française (Sechehaye 1926a)

Première partie :

Groupe substantif. Substantif, déterminatifs, pronoms absolus, adjectif attribut, adverbes de l'adjectif. 
Chap. I : Le substantif

Chap. II : Les articles

Chap. III : Les autres déterminatifs du substantif

Chap. IV : Les noms de nombre

Chap. V : Les pronoms

Chap. VI : L'adjectif attribut

Deuxième partie :

Le groupe verbal. Verbe à l'indicatif, adjectif et substantif prédicatifs, adverbes du verbe.

Chap. VII : Le verbe et son sujet

Chap. VIII : Le verbe à l'indicatif

Chap. IX : L'adjectif et le substantif prédicatifs

Chap. X : L'adverbe du verbe

Troisième partie :

Le complément de relation. Substantif ou pronom complément avec ou sans préposition.

Chap. XI : Le complément sans préposition

Chap. XII : Le complément prépositionnel

Chap. XIII : Déterminatifs et pronoms absolus compléments

Chap. XIV : Les pronoms conjoints compléments

Chap. XV : Le verbe réfléchi et le verbe pronominal

Chap. XVI : Prédicat dans le complément direct

Chap. XVII : Complément de comparaison

Quatrième partie :

Les formes nominales du verbe. Infinitif, participes, gérondifs et constructions absolues.

Chap. XVIII : L'infinitif

Chap. XIXa : Les participes

Chap. XIXb : Les constructions absolues

Cinquième partie :

Généralités sur la proposition indépendante à l'indicatif. Coordinations. Les propositions indépendantes modales : conditionnel, subjonctif, impératif, interrogation, exclamation.

Chap. XX : Les propositions indépendantes à l'indicatif

Chap. XXI : Le conditionnel modal

Chap. XXII : L'impératif 
Chap. XXIII : Le subjonctif exprimant l'ordre et le souhait

Chap. XXIV : L'interrogation

Chap. XXV : Exclamation et interjections

Appendice : La ponctuation dans les propositions indépendantes et dans les coordinations de propositions

Sixième partie :

Les propositions subordonnées : relative, interrogation indirecte, complétive, circonstancielles, incidente.

Chap. XXVI : La proposition relative

Chap. XXVII : L'interrogation indirecte

Chap. XXVIII : La complétive

Chap. XXIX : Les circonstancielles

Chap. XXX : La concordance des temps

Chap. XXXI : Les incidentes

Appendice : La ponctuation dans les phrases qui contiennent des subordonnées

Index

Tableau des conjugaisons

Annexe II

Les phrases (énoncés) types dans l'Abrégé

Groupe substantif :

Cet élève, celui-ci, ce très bon élève.

Groupe verbal :

Cet élève travaille, a travaillé, travaillera.

Ce travail est mauvais; il reste beaucoup de fautes.

Il ne travaille pas consciencieusement.

Le complément de relation :

Paul a fait un travail pour l'école. Le travail de Paul est bon. Le maître loue Paul; il le loue. Il est content de son travail ; il en est content.

Paul se réjouit : son travail est meilleur que celui de Pierre.

Les formes nominales du verbe :

Apprendre le français est difficile. Il est difficile d'apprendre le français sans maître. Cette leçon est difficile à apprendre. J'ai à peine le temps de l'apprendre. Je vais essayer de l'apprendre. J'ai rencontré Paul tenant un livre ouvert. Il apprend le français en lisant beaucoup. Un livre fini, il en commence un autre.

Généralités sur la proposition indépendante à l'indicatif. Coordinations. Les propositions indépendantes modales : 
Le maître explique aux élèves une règle importante, mais difficile. Aussi tous sont-ils attentifs. On entendrait voler une mouche dans la classe. "Suivez bien mes explications. Que chacun fasse attention. As-tu compris, Paul ? - « Non? Qu'est-ce que tu as compris? » « Ah! voilà une question intelligente, bravo!»

\section{Les propositions subordonnées :}

L'examen que vous avez à subir sera difficile. Vous ne pouvez pas savoir sur quoi vous serez interrogé. Il importe donc que vous connaissiez à fond tous les sujets. Si vous aviez travaillé régulièrement pendant l'année, vous n'auriez rien à craindre. Mais vous êtes en retard parce que vous avez été trop souvent absent. À peine vous revoyait-on à l'école que vous disparaissiez de nouveau. Je sais que vous travaillez bien, mais « Rien ne sert de courir, dit le poète, il faut partir à temps ».

\section{NOTES}

1. * Le présent texte constitue une version remaniée de la conférence plénière faite au colloque de Raguse, le 8 juin 2012. Je tiens à remercier Henri Besse, Jean-Claude Chevalier, Enrica Galazzi et Nadia Minerva de leurs remarques, et Annette Fryba pour tout ce qu'elle m'a appris à propos de Sechehaye. Mes remerciements vont également à la rédaction et au secrétariat de la revue L'Éducateur (Nicole Rohrbach et V. Jacques Darbellay) pour la documentation mise à ma disposition.

Sur ce type (récurrent) de querelles, cf. Swiggers (1992).

2. Albert Chessex (né en 1881 à La Chiésaz) fut instituteur à Brenles-sur-Moudon et à Pully, maître primaire supérieur à La Sarraz et à Lausanne, et termina sa carrière comme maître de pédagogie pratique à l'École normale et comme directeur du musée scolaire cantonal dans le Vaud. Patoisant vaudois et écrivain, il a collaboré avec E. Schüle au Petit dictionnaire vaudois (français-patois) (1963); parmi ses ouvrages littéraires, on peut mentionner Les Alpes Vaudoises (1949) et Par monts et vaux: promenades et excursions (1952). Il est le co-auteur, avec Henri Jeanrenaud, d'un manuel de Didactique générale (1943). Sur son importance comme pédagogue, voir Forster (2004).

3. Il est intéressant à cet égard de citer l'ouverture du compte rendu de Henri Yvon à propos du même ouvrage : «Le livre de M. Sechehaye ne peut, ce qui est fort regrettable, être utilisé dans l'enseignement en France : l'auteur emploie en effet la terminologie grammaticale en usage dans les pays de langue allemande. Ce petit fait amène à souhaiter fortement qu'il s'établisse, au moins pour les peuples de l'Europe occidentale, dont les langues présentent tant de ressemblances entre elles, une nomenclature commune. [...] De bons esprits estiment que ce serait une tâche intéressante pour l'Institut de Coopération intellectuelle, de chercher une entente à ce sujet. Toutefois, au moment même où nous formulons ce souhait, une vois décourageante nous dit que nous ne le verrons sans doute pas se réaliser : nous savons que seize ans après sa promulgation, une nomenclature simplifiée, établie pour répondre aus voeus du personnel enseignant, n'est pas encore pleinement en usage dans notre pays. À quelles résistances, à quelle inertie plus redoutable encore que les résistances se heurtera-t-on si l'on propose prochainement des modifications à cette nomenclature à peine admise? Pour ne citer qu'un détail, l'opposition est complète, en ce qui concerne l'adjectif entre les termes épitète, attribut, employés en France, et le couple attribut, prédicat, employé à l'étranger. Au nom de quel principe obtiendra-t-on que tout un personnel enseignant renonce à des expressions ausquelles il s'est accoutumé, peut-être non sans difficultés »? (Yvon 1927 : 144-145) [nous avons respecté la graphie du texte original]. 
4. Conflit que Sechehaye explicite de manière très nette : «Le mal dont souffre l'enseignement grammatical a sa source dans ce fait qu'on a toujours paru penser qu'il suffisait, pour enseigner une langue, de la posséder et d'user d'une certaine habileté pratique. Encore aujourd'hui, la plupart des efforts qui tendent à améliorer la méthodologie s'occupent avant tout du problème psychologique et pédagogique et pas ou très peu du problème linguistique. On veut savoir comment il convient d'enseigner une langue à des enfants et pour cela on cherche à connaître l'esprit de l'enfant, en quoi on a raison, mais on ne se demande pas ce que c'est qu'une langue, en quoi on a tort. Une langue ne se décrit pas comme on décrit un objet matériel quelconque, un ensemble de lignes, de formes et de couleurs; une langue est un organisme qui fonctionne d'une certaine façon; on ne saurait la décrire sans posséder les connaissances techniques indispensables. N'importe qui peut décrire une montre, mais sans certaines connaissances de mécanique cette description restera nécessairement insuffisante, superficielle et verbale. Or, c'est précisément ce qui arrive à la grammaire traditionnelle quand elle parle des faits de langue, faute d'avoir essayé de pénétrer dans leur vraie nature. Elle nomme les choses sans les bien connaître, sans les analyser, sans les distinguer correctement. Cette grammaire qui s'inspire d'une méthode tout empirique s'est édifiée à travers le temps pour répondre aux besoins d'un enseignement qui ne visait qu'à un résultat pratique et formel : la correction du langage et spécialement la correction orthographique. Au cours de sa longue carrière, elle a subi une sorte de polissage et acquis une certaine perfection extérieure qui peut donner le change, mais qui masque mal à un regard scrutateur ses insuffisances. Son plus grand tort, c'est peut-être d'avoir fait naître chez les pédagogues qui se confient en elle une profonde insouciance des problèmes qu'elle ne résout pas mais qu'elle dissimule. » (Sechehaye 1927 : 14-15)

5. Pour des données biographiques, voir Lázaro-Carreter (1947), Reverdin (1959), Wiblé (1946-47).

6. Pour une bibliographie des travaux linguistiques de Sechehaye, voir AN. (1945) [liste sommaire, sans doute établie par l'auteur lui-même] et Fryba-Reber (1994: 183-188). Ce dernier travail fournit une analyse détaillée des conceptions linguistiques de Sechehaye, surtout à partir de son Essai sur la structure logique de la phrase ; voir aussi Fryba-Reber (1995-96).

7. Dans une lecture à rebours et "de complicité circonstancielle", on pourrait parler d'une poussée schizophrénique ; rappelons que Mad. Sechehaye (Marguerite Jenny Sechehaye-Burdet) était une autorité dans le domaine de l'approche et de la thérapie de la schizophrénie (cf. ses livres La réalisation symbolique, 1947; Journal d'une schizophrène, 1950; Introduction à une psychothérapie des schizophrènes, Paris, 1954).

8. Thèse publiée, en français, sous forme d'un long article (Sechehaye 1905 ; cf. le compte rendu de Yvon 1905).

9. Mad. Sechehaye avait suivi le troisième (et dernier) cours de linguistique générale de Saussure ; $c f$. Fryba-Reber (1994 : 11). Selon la formule fort apte de la notice nécrologique publiée dans les Cahiers Ferdinand de Saussure 5 (1945), Sechehaye fut « disciple de Ferdinand de Saussure sans avoir été son élève ».

10. Comme le signale Jakobson (1939), Sechehaye s'est intéressé très tôt à des problèmes de phonologie. Il a d'ailleurs enseigné pendant longtemps un cours de « Phonologie " (cf. infra, note 25); cf. aussi son compte rendu de L. Michel, Tendances de la linguistique contemporaine: la phonologie [1942], dans Cahiers Ferdinand de Saussure, 5 (1945), 70-71.

11. «Symbolique et syntaxe dans leur ensemble, la première emboîtant la seconde, représentent la Grammaire, qui s'occupe de tous les faits d'expression conventionnelle. La Grammaire, elle, est emboîtée dans la psychologie. Elle représente un aspect acquis, artificiel, pour ainsi dire, de notre vie psychophysique individuelle, qui reste le fond indispensable et, en dernière analyse, toujours le facteur le plus puissant de notre langage. [...] La grammaire, cette organisation du langage, nous apparaît donc comme étant par sa nature et l'œuvre et l'instrument de la pensée humaine. Notre langage est supérieur à celui des animaux en perfection grammaticale, parce que nous leur 
sommes supérieurs en intelligence. [...] La grammaire, symbolique et syntaxe, est donc une institution intellectuelle en perpétuel flottement. » (Sechehaye 1914 : 290-291)

12. Voir aussi Sechehaye (1917).

13. Cf. le passage suivant: "L'une consiste à partir du système des formes (formes fléchies, groupes de mots, etc.), et à étudier leurs valeurs et leurs emplois. L'autre, au contraire, voudrait qu'on posât d'abord le système des éléments psychiques de la langue pour rechercher ensuite les formes concrètes de leur expression. » (Sechehaye $1916: 45)$

14. Auteur de l'Enseignement régulier de la langue maternelle et du Cours éducatif. Voir aussi le texte de la conférence sur "La méthode constructive en grammaire et son application » (Sechehaye 1930 réimpr. 1944 : 23-25) : «c'est [...] le père Girard (1765-1830) qui a formulé le principe sur lequel une grammaire bien ordonnée peut s'établir: il faut prendre la phrase et non le mot comme point de départ du système grammatical. » (ibid. : 23)

15. Elle englobe différents phénomènes: ellipse; inversion; usage d'équivalents de phrase ; modalisation de l'énoncé (supposition; probabilité) ; marquage formel des modalités ou de la polyphonie.

16. C'est un concept auquel Sechehaye restera attaché ; $c f$. Sechehaye (1941: 79).

17. Dans le travail commun de Bally et Sechehaye présenté au premier Congrès international des linguistes (1928) les auteurs insistent davantage sur l'irréductibilité de cette opposition ; $c$. Bally et Sechehaye (1928: 37 et 48) : « la grammaire, qui ne peut être que statique, se ramène toute à l'étude systématique des procédés d'expression, tandis que l'histoire étudie les procès qui altèrent ou détruisent ces procédés »), mais ils reconnaissent que «l'histoire pourrait rentrer dans la statique par une porte dérobée " (ibid.: 41: il s'agit des cas de flottement ou de concurrence entre syntagmes).

18. Cf. le texte de la conférence sur "La méthode constructive en grammaire et son application » (Sechehaye 1930 réimpr. 1944: 25): «Dans l'application pratique, il ne s'agit pas d'un plan rigoureux et rigide. La méthode s'accommode avec souplesse aux exigences d'un enseignement élémentaire, tant en ce qui concerne la langue maternelle qu'en ce qui concerne les langues étrangères. Elle n'apparaît dans toute sa rigueur que lorsqu'il s'agit de tirer des conclusions générales de l'enseignement grammatical, ce qui est le fait des classes supérieures. Elle est avant tout un esprit qui inspire le pédagogue et qui le porte à s'attacher à des objets concrets vus dans leur fonctionnement. Cette méthode, en un mot, répond aux besoins d'un enseignement vivant et pratique, véritablement moderne, tandis que la méthode traditionnelle est l'expression naturelle de la grammaire ancienne, scolastique et formelle. C'est un anachronisme que de s'attacher à elle. »

19. Sechehaye (1920); dans ce travail il expose sa conception à propos de la phrase-idée et de la phrase-pensée. Ce texte est tiré d'un long manuscrit, rédigé vers 1913, que Sechehaye laissera mûrir pendant plus de dix ans, avant de lui donner sa forme définitive dans son Essai sur la structure logique de la phrase (Sechehaye 1926b).

20. Brunot (1920-21) parle de « renouvellement (nécessaire)».

21. Voir l'étude détaillée de Lauwers (2004). Pour les manuels publiés en Allemagne, voir Niederländer (1981).

22. Pour une justification de cette jonction harmonieuse, couplée avec un appel habile à l'autorité de Saussure, voir Bally et Sechehaye (1928: 53) : «En linguistique, comme dans toute science, la pratique, pour être féconde, doit s'inspirer de principes solides, et ceux-ci ne peuvent être saisis, définis et classés que par un effort d'abstraction. D'aucuns peuvent penser que l'école qui se réclame de F. de Saussure accorde trop de place à la spéculation; mais on ne doit pas oublier que dans ses conceptions les plus générales et les plus abstraites, elle vise des fins éminemment pratiques, et quiconque s'efforcera d'asseoir la science du langage sur une base théorique irréprochable sera par là même assuré de la rendre plus accessible et plus apte aux réalisations concrètes. » 
23. Cf. Fryba-Reber (1994: 179) : «Le mérite de Sechehaye ne serait-il pas en définitive d'avoir compris à travers l'exemple de Saussure que théorie et pédagogie constituaient au fond deux faces indissociables de toute entreprise linguistique? Qu'en linguistique tout au moins, la pédagogie, loin d'être un simple mode d'exposition de la théorie, fait en réalité partie intégrante de la conceptualisation elle-même? Toute la recherche de Sechehaye aura été à cet égard de relever ce défi. »

24. $C f$. aussi le témoignage suivant de Sechehaye, dans sa première étude de syntaxe historique, sur sa volonté de comprendre l'évolution de la langue: "Il ne s'agit point ici de lois mathématiques, amenant fatalement un résultat calculable d'avance, car l'arbitraire humain est un des facteurs essentiels de ces évolutions. Cependant cet arbitraire ne marche pas au hasard et au gré d'une fantaisie capricieuse. Euvre d'une collectivité, la langue se soustrait moins facilement à un certain déterminisme que si elle était la création d'un seul individu. Aussi la science qui mesure à peu près la force des influences en lutte, et qui dit quelles sont celles qui, étant suivies, fourniront à la langue des moyens d'exprimer la pensée plus simplement, plus clairement et plus complètement, apportera-t-elle toujours quelque lumière pour faire comprendre le pourquoi des évolutions syntactiques. » (Sechehaye 1905 : 382)

25. Dans l'enseignement de Sechehaye à Genève ( $c f$. le relevé de ses cours dans Fryba-Reber 1994 : 194-205), on note la présence continue d'un cours de grammaire historique (de 1904 à 1946 !), d'un cours de « Stylistique » (de 1918 à 1940). Entre 1903 et 1905 Sechehaye a fait un cours de français préclassique et de 1909 à 1914 un cours de «Phonologie ». À partir de 1930 il s'est consacré à la grammaire synchronique du français et à son enseignement, comme en témoignent les cours de "Grammaire constructive» (1930-1931), "Questions pratiques concernant la grammaire constructive (1938 à 1940), « Théorie et méthodologie de la grammaire (1931 à 1935) et «Questions pratiques concernant l'enseignement de la grammaire française " (de 1940 à 1942). De 1934 à 1945 Sechehaye a été chargé du cours d' «Introduction à la science du langage ».

26. Tout comme Antoine Grégoire (1927: 313) : «[...] si restreinte que soit la destination de l'ouvrage, la lecture n'en est pas moins recommandable à tous les professeurs de grammaire ; car il est conçu d'après un plan nouveau, propre à l'auteur, qui en a exposé jadis les principes dans la Revue des langues romanes, en 1916. Ce plan rompt avec la disposition traditionnelle des matières grammaticales. Non seulement il en devient plus pratique, mais il a le grand mérite de refléter les faits du langage plus exactement et plus clairement. "

27. Le philosophe du quotidien tirera la morale de l'histoire (/de cette histoire) : en linguistique, et encore moins en didactique des langues, il est difficile, voire impossible, de contenter tout le monde et sa belle-mère.

28. D'autant plus que Chessex répliqua dans les termes suivants : "Merci à M. Sechehaye de sa courtoisie. Mais au risque de lui déplaire une seconde fois, je dois lui dire que sa réponse ne m'a nullement convaincu. Mon contradicteur continue à ne pas faire la distinction - capitale à mon sens - entre le savant et l'écolier. S'il s'agissait de gymnasiens ou d'étudiants, rompus au jeu des idées et familiarisés avec les abstractions, rien ne s'opposerait à ce qu'on leur présentât telle quelle la grammaire de M. Sechehaye ; mais pour des écoliers de 12 à 15 ans, je maintiens qu'il faut viser à être avant tout simple et pratique. Tout autant que M. Sechehaye, je suis soucieux de comprendre et de faire comprendre ; mais les langues offrent un champ suffisant à la réflexion et à l'intelligence, sans qu'il soit nécessaire de proposer à l'enfant des subtilités qui lui passent pardessus la tête. Je ne vois pas, par exemple, - et en dépit des explications de M. Sechehaye, - la nécessité du terme spécial de complément partitif conjoint. Si l'essentiel est de comprendre, je ne discerne pas ce que l'introduction d'un terme nouveau pourra faire gagner à la compréhension. Je vois fort bien au contraire la confusion et la lassitude qui résulteront presque fatalement de la surabondance et de la complication de la nomenclature. Il va sans dire que je ne mets pas en doute la science de M. Sechehaye. Je prétends seulement que le spécialiste qui se fait maître d'école doit garder pour soi une bonne partie de son savoir.» (Chessex 1927 : 16) 


\section{RÉSUMÉS}

L'Abrégé de grammaire française sur un plan constructif (1926) d'Albert Sechehaye est un ouvrage à orientation didactique qui est en rapport direct avec ses conceptions de linguiste théoricien : l'auteur y expose (ses vues sur) la structuration progressive de la matière linguistique telle qu'elle doit être apprise graduellement par l'élève. C'est à travers la présentation de phrasestypes, qui reflètent une complexité syntactico-discursive croissante et qui s'appuient sur les opérations de substitution et d'expansion que l'apprenant saisit le mécanisme formateur de la langue et qu'il apprend à construire le "système de la grammaire ». L'article présente une analyse des conceptions théoriques de Sechehaye et de leur mise en application didactique dans leur rapport avec l'attachement à la linguistique saussurienne ; la réception, immédiate et en moyenne durée, de l'œuvre didactico-linguistique de Sechehaye est également étudiée.

The Abrégé de grammaire françaisesur un plan constructif (1926) of Albert Sechehaye is a didactically oriented work closely tied up with the author's conceptions as a theoretical linguist; in this work Sechehaye exposes (his views on) the progressive structuring of the language material as it has to be gradually mastered by the pupil. Through the presentation of sentence types (phrases-types), which reflect the gradually increasing syntactic-discursive complexity and which are based on the operations of substitution and expansion the learner grasps the formative mechanism of a language and learns to construe the 'system of grammar'. The article offers an analysis of the theoretical conceptions of Sechehaye and their didactic implementation in relation to their close connection with Saussure's linguistics; the reception of Sechehaye's linguistic-didactic contribution is also studied.

\section{INDEX}

Mots-clés : didactique du français, École de Genève, grammaire constructive, grammaire française, phrases-types, Sechehaye, structuralisme

Keywords : constructivist grammar, French grammar, Geneva School, Sechehaye, sentence types, structuralism, teaching of French

\section{AUTEUR}

\section{PIERRE SWIGGERS}

K.U. Leuven \& Université de Liège, Belgique

Pierre.Swiggers@arts.kuleuven.be 